Miranda Contreras, B. E., y Villarreal Amarís, G. E. (enero-abril, 2022). Efecto de un programa de promoción y prevención sobre la comunicación y lenguaje de escolarizados en instituciones públicas de Sincelejo. Revista Virtual Universidad Católica del Norte, (65), 171-

199. https://www.doi.org/10.35575/rvucn.n65a7

\title{
Efecto de un programa de promoción y prevención sobre la comunicación y lenguaje de escolarizados en instituciones públicas de Sincelejo
}

\author{
Effect of a promotion and prevention program on the communication and language of \\ schoolchildren in public institutions of Sincelejo
}

\section{Beatriz Elena Miranda Contreras \\ Magister en Neuropsicología \\ Facultad Ciencias de la Salud, Universidad de Sucre \\ Sincelejo, Colombia \\ beatriz.miranda@unisucre.edu.co}

Orcid: https://orcid.org/0000-0001-8105-784X

CvLAC:

https://scienti.minciencias.gov.co/cvlac/visualizador/generarCurriculoCv.do?cod rh=0000692999

\section{Gloria Esther Villarreal Amarís}

Magister en Salud Pública

Facultad Ciencias de la Salud, Universidad de Sucre

Sincelejo, Colombia

gloria.villarreal@unisucre.edu.co

Orcid: https://orcid.org/0000-0003-4169-139X

CvLAC:

https://scienti.minciencias.gov.co/cvlac/visualizador/generarCurriculoCv.do?cod rh=0000318981

Recibido: 15 de febrero de 2021

Evaluado: 07 de octubre de 2021

Aprobado: 19 de enero de 2022

Tipo de artículo: Investigación Científica y Tecnológica

\section{Resumen}

El objetivo del estudio fue identificar y analizar cambios a nivel comunicativo y lingüístico de estudiantes vinculados al Proyecto "Salud Escolar", implementado en entornos escolares, para 
población de 6 a 17 años. Metodológicamente se realizó un estudio cuantitativo, no experimental, alcance descriptivo, longitudinal (retrospectivo). Los instrumentos utilizados fueron la encuesta y revisión documental, los cuales permitieron identificar al programa "Juguemos con los sonidos" con mayor continuidad; en la dimensión "efectividad del aprendizaje", todos los encuestados reportaron dificultades en el desempeño y habilidades escolares de los niños y niñas, antes de vincularse al proyecto, con puntuaciones porcentuales comprendidas entre (máx. $=99 \% ; \mathrm{D}=18,2)$ a (máx. $=30 \% ; \mathrm{D}=9,8 \%$ ); pero, después de vinculados al proyecto estas puntuaciones mejoraron (máx.=95\%; $\mathrm{D}=14,5 \%)$ a (máx.=90\%; D=10\%). Para la dimensión "capacidades de desempeño adquirido", todos los participantes coincidieron en que las habilidades alcanzadas son: comunicación oral, conciencia fonológica y procesos de lectoescritura, aunque docentes y administrativos de instituciones educativas incluyen también comprensión lectora, buenos hábitos orales y el autocuidado de la audición, con porcentajes obtenidos entre $50 \%$ y $100 \%$. En conclusión, se pueden observar cambios en habilidades comunicativas y lingüísticas de estudiantes, después de implementarse estrategias de promoción y prevención en el entorno escolar.

Palabras Clave: Efectos sobre la comunicación; Escolar; Evaluación de proyecto; Prevención; Promoción; Salud.

\begin{abstract}
The objective of the study was to identify and analyze changes at the communicative and linguistic level of students linked to the "School Health" Project, implemented in school environments, for a population aged 6 to 17 years. Methodologically, a quantitative, non-experimental study, descriptive, longitudinal (retrospective) scope was carried out. The instruments used were the survey and documentary review, which made it possible to identify the program "Let's play with sounds" with greater continuity; In the dimension "learning effectiveness", all respondents reported difficulties in the performance and school skills of boys and girls, before joining the project, with percentage scores between $(\max .=99 \% ; \mathrm{D}=18.2)$ a $(\max =30 \% ; \mathrm{D}=9.8 \%)$; But, after being linked to the project, these scores improved $(\max =95 \% ; \mathrm{D}=14.5 \%)$ to $(\max =90 \%$; $\mathrm{D}=10 \%$ ). For the dimension "acquired performance skills", all participants agreed that the skills
\end{abstract}


achieved are: oral communication, phonological awareness and literacy processes, although teachers and administrators of educational institutions also include reading comprehension, good oral habits and self-care of hearing, with percentages obtained between 50\% and 100\%. In conclusion, changes in communication and linguistic abilities of students can be observed, after implementing promotion and prevention strategies in the school environment.

Key Words: Effects on communication; School; Project evaluation; Prevention; Promotion; Health.

\section{Introducción}

El entorno escolar es considerado uno de los ambientes más importantes en el desarrollo integral del ser humano (Castro y Morales 2015). Por tal motivo, al acompañar actividades de enseñanza, con estrategias integrales de aprendizaje, complementada esta con una adecuada atención y fortalecimiento de todas las áreas de desarrollo (motor, cognitivo, personal-social, adaptativo y comunicativo) del infante y adolescente, se reflejará a futuro en un adecuado avance del ser humano. Por lo anteriormente expuesto, se considera que desde las instituciones de educación deben surgir iniciativas que vayan en pro de fortalecer las rutas de atención integral de estudiantes, desde una perspectiva de derechos, ahondando en aspectos que permitan el desarrollo pleno de niños y niñas (Política Nacional de Infancia y Adolescencia 2018-2030).

Por tal motivo, es importante que la población infantil y juvenil acceda a servicios de salud integrados. Estos servicios de salud integrales, según la Organización Panamericana de la Salud OPS- y la Organización Mundial de la Salud -OMS- (2010) se consideran como:

La gestión y prestación de servicios de salud de forma tal que las personas reciban un continuo de servicios de promoción, prevención, diagnóstico, tratamiento, gestión de enfermedades, rehabilitación y cuidados paliativos, a través de los diferentes niveles y sitios de atención del sistema de salud, y de acuerdo a sus necesidades a lo largo del curso de vida. (p. 29)

Por otro lado, para la OPS y OMS (2010) es de gran importancia una atención primaria en salud renovada, cuya característica principal se enfoque en atender a las poblaciones más 
necesitadas, ofreciéndoles servicios permanentes, bien planeados, y buscando siempre la participación activa de todos los involucrados en los procesos, bajo la intervención coordinada de instituciones representativas de diferentes sectores sociales, que tengan como base hacer que esas comunidades en situación de subdesarrollo alcancen un adecuado bienestar social y calidad de vida.

Así las cosas, se considera de gran importancia destacar que, desde la educación, los temas relacionados con salud es importante abordarlos desde edades tempranas, teniendo en cuenta tanto el contexto familiar como el escolar, constituyéndose la escuela como ese espacio propicio para inculcar en los niños y niñas hábitos saludables (Florián, 2019, p. 130). En otras palabras, se puede considerar al colegio como uno de los elementos fundamentales en el desarrollo de la niña y el niño, en tanto juega un papel fundamental en la elaboración de aquellos saberes y habilidades que permiten la toma de decisiones saludables, relacionadas con el autocuidado de su salud y la salud de los que le rodean.

Asi pues, existen personas que asumen comportamientos no saludables, lo cual conlleva a atentar contra su salud, y podrían ser considerados como factores de riesgo para el padecimiento, a futuro, de enfermedades; en el caso contrario, existen personas que asumen comportamientos saludables, conllevándolos a no presentar problemas en su salud. Este tipo de comportamientos son los que van a establecer los estilos de vida de los individuos. Por tal motivo, se debe preparar a los niños y a las niñas, desde etapas tempranas, para que adquieran y moldeen conductas saludables, a través de la educación y promoción en salud, logrando así que estas perduren a lo largo de la vida (Bennasar, 2011).

En este sentido, la Facultad de Ciencias de la Salud de la Universidad de Sucre (UNISUCRE), la cual la conforman los departamentos de Fonoaudiología, Enfermería y Medicina, ha venido implementando estrategias de trabajo comunitario, que conjugan los tres ejes misionales de la Universidad: docencia, prestaciones de servicios e investigación, desde su programa de Extensión y Proyección Social, lo que ha permitido su posicionamiento como institución de educación superior con compromiso social. El programa bandera -programa comunitario de la Facultad- inicia después de firmado el convenio con la Alcaldía municipal en 1996; este facilitó fortalecer los vínculos de docencia y servicio, y posibilitó el adelanto de acciones de atención en 
salud y desarrollo comunitario en poblaciones con características de marginalidad y extrema pobreza de la región.

Dentro del programa de proyección social de la Facultad se encuentra el proyecto "Salud escolar", cuyo escenario de acción son las escuelas de los barrios de la zona suroriental de la ciudad de Sincelejo, Colombia. El proyecto tiene como objetivo: "desarrollar acciones orientadas a la valoración del desarrollo físico, mental y social del escolar, con el fin de fomentar hábitos higiénicos y comportamientos saludables, desde su entorno escolar y familiar" (Villareal, 2012). Así las cosas, desde el accionar fonoaudiológico se viene trabajando, con el apoyo de docentes y estudiantes en formación, los tres subproyectos que de este se desprenden, como son: Juguemos con los sonidos, el cual va dirigido a estudiantes de transición y de los grados primero y segundo de Educación Básica Primaria; el subproyecto Leo, escribo y aprendo, el cual va dirigido a los estudiantes de los grados tercero, cuarto y quinto de Educación Básica Primaria; y el subproyecto Comuniquémonos mejor, que va dirigido a los estudiantes de Educación Básica Secundaria, para así contribuir con la mejora permanente de la calidad auditiva, comunicativa, del lenguaje y del aprendizaje de menores y adolescentes en condiciones de vulnerabilidad del municipio, siendo abordadas desde el ambiente escolar, a través de actividades de promoción, prevención (desde los niveles uno y dos de atención), consultoría, asesoría y evaluación, las cuales son asumidas con compromiso y respeto a lo estipulado en las políticas de desarrollo nacional, que buscan propender por el beneficio de los usuarios y de la comunidad en general.

Igualmente, el programa viene contemplando el desarrollo de investigaciones que den cuenta de resultados efectivos, los cuales transformen políticas en metas específicas y acciones necesarias, permitiendo así la generación de nuevo conocimiento en acciones de promoción y prevención de la salud comunicativa. Es así como el Consejo de Facultad Ciencias de la Salud de la Universidad de Sucre expide la Resolución 022 de 2019, donde:

Avala y recomienda ante el centro de investigaciones de la Universidad de Sucre (DIUS), la ejecución del proyecto titulado Experiencias académicas del programa de Fonoaudiología de la UNISUCRE en prácticas comunitarias de la Zona suroriental de Sincelejo, durante el período 2013-2017.

Dicho proyecto tuvo como objetivo general: evaluar el efecto del Programa Salud Escolar, durante los años 2013-2017, sobre la comunicación y lenguaje de estudiantes de la Zona 
Suroriental, Sincelejo-Colombia. Además, como objetivos específicos se buscó: i) establecer el grado de participación en el programa Salud Escolar de estudiantes y docentes de las instituciones educativas, y estudiantes, egresados y docentes de la UNISUCRE durante el período 2013-2017; ii) determinar el efecto en las capacidades de desempeño adquiridas, de nivel cognitivo y comunicativo, de niños y niñas involucrados en el proyecto; y iii) identificar el efecto en la actitud hacia procesos de aprendizaje, porcentajes de efectividad del aprendizaje y posibilidad de cambio en el desempeño de los involucrados en el proyecto.

El logro de los objetivos programados permitió al programa de Fonoaudiología de la UNISUCRE determinar si las estrategias y actividades implementadas en el desarrollo del proyecto, con sus subproyectos, promueven y fomentan entornos comunicativos saludables que tributen en el mejoramiento del aprendizaje escolar, y en la participación activa de los diferentes actores (docentes y directivos, entre otros profesionales) involucrados en el proceso. Igualmente, contribuyó en la generación de compromiso y corresponsabilidad, dentro de las instituciones educativas, con la comunidad en general de la zona. También, permitió determinar si la creación e implementación de los programas en el proyecto cumplían con las necesidades reales de este sector de la población, de modo que el impacto social que se quiere lograr sea la adopción de estilos comunicativos saludables, desde edades tempranas, con la ejecución correcta de los programas. Igualmente, permitió al programa dar cumplimiento a las a recomendaciones dadas por el Consejo Nacional de Acreditación (CNA), en su visita para acreditación del programa en alta calidad; como también, reorientar los procesos de enseñanza - aprendizaje, logrando una adecuada relación entre lo teórico y lo práctico, la investigación y la intervención, lo que posibilitó el estar constantemente autoevaluando los procesos.

\section{Referentes investigativos y teóricos}

Dentro de los referentes investigativos se hace referencia a diversos estudios internacionales y nacionales, como los relacionados a continuación. En España, Ramos et al. (2016) describieron el desarrollo del programa de Salud Escolar pedagógica durante los años 2004 a 2015 (estudio de 12 años), con el que buscaron valorar tempranamente los signos de enfermedad que se podían manejar tempranamente, e igualmente identificar factores de riesgo pedagógico 
prevenibles a través de programas de educación en salud. Formaron parte del estudio, la comunidad escolar de diferentes centros de enseñanza de primaria y secundaria, y los responsables de las prácticas de la asignatura Pedagogía Preventiva y Comunitaria, llegando a explorar un total de 4.630, de los cuales 677 presentaban alteraciones pedagógicas. También, se observó que se benefició a 7.145 miembros de la comunidad escolar, teniendo un acercamiento con la población a través de actividades educativas sobre el autocuidado, con el objetivo de prevenir dificultades posteriores que afectaran la calidad de vida (Ramos et al., 2016).

Silva et al. (2018), por otro lado, realizaron un estudio cualitativo de la práctica fonoaudiológica en el contexto de la atención primaria de la salud, en la quinta región de Valparaíso, llegando a concluir que la academia debe responsabilizarse de la orientación y formación enfocada en las necesidades actuales del sistema de salud, en lo relacionado con la atención primaria, buscando abarcar de esta manera todo lo relacionado con lo pedagógico y lo comunitario, reduciendo gradualmente el abordaje terapéutico-asistencial con el que se viene trabajando desde la profesión. Dejan por sentado que para el abordaje comunitario se debe contar con una cultura de servicio, cuya responsabilidad va a recaer sobre el profesional de la salud.

En otro estudio realizado por Arredondo Kassabb (2019), titulado: Buenas prácticas socioeducativas comunitarias para la atención logopédica en la comunicación y el lenguaje de niños y niñas en etapa escolar, adolescentes, jóvenes y adultos, se concluye que se deben elaborar estrategias encaminadas a la prevención y estimulación del lenguaje y la comunicación de poblaciones de diferentes ciclos vitales. Igualmente, exhorta la necesidad de ir pensando nuevas opciones de intervención del desarrollo comunicativo y del lenguaje que favorezcan habilidades sociales e interpersonales, de una manera permanente, en los diferentes grupos poblacionales.

Por otro lado, la evidencia científica informa que, en el Reino Unido, los niños que reúnen los requisitos para recibir comidas escolares gratuitas y que viven en barrios desfavorecidos, presentan en 2,3 la probabilidad de ser identificados con problemas de habla, lenguaje y comunicación (Dockrell \& Marshall, 2014, Henwood et al., 2020). Por su parte, Waldfogel y Washbrook (2010) manifiestan que los niños que asisten a las escuelas, provenientes de familias con bajos ingresos, están en desventaja, casi un año, en comparación con los niños de ingresos familiares más altos, en términos de desarrollo del vocabulario (p. 7). 
En Colombia, Carreño Patiño (2018) realizó un estudio titulado: Acciones del fonoaudiólogo(a) en el marco de la Atención Primaria de Salud (APS); la teoría frente a la práctica. En él, concluye que son dos las posibilidades con las que cuenta el profesional en fonoaudiología para su desempeño profesional, una es la asistencial y la otra es la administrativa.

En cada una de ellas, el profesional toma elementos propios de su cuerpo de conocimientos y de su práctica, tal como lo resalta la conferencia de Alma-Ata científicamente fundados y socialmente aceptables, con el objetivo de garantizar un acceso a los servicios de salud de todos los individuos y familias de las comunidades a un costo razonable. (p. 54)

También, en el estudio titulado “Comprensión de las Prácticas Fonoaudiológicas realizadas por egresados del programa de Fonoaudiología de la Corporación Universitaria Iberoamericana con la aplicación del modelo Sistémico de la comunicación”, Cortés et al. (2017), finalizando la investigación, determinaron que:

Los egresados (...) entre los años 2006 al 2016 ejecutan funciones de asesoría, consejería, consultoría, promoción y prevención de la salud comunicativa, evaluación- diagnóstico e intervención, en escenarios de bienestar social y comunitario, educativo y clínico, en donde ejercen roles tales como: asistencial, docente, administrativo e investigativo, (...) generando una identidad institucional y siendo reconocidos por su abordaje integral. (p. 49)

De otro lado, dentro de los referentes teóricos que sustentan el artículo se describen diferentes conceptos como comunidad, cuyo origen se determina a partir del inicio de la existencia de los seres humanos. Son muchas las definiciones que se pueden encontrar relacionadas con comunidad, y que tienen en cuenta diferentes posiciones y abordajes. Según la concepción de comunidad, que se elaboró en los trabajos de la Comisión Ministerial para el Trabajo Comunitario Integrado de Cuba, es definida por Reyes y Góngora (2010) como:

El espacio geográficamente delimitado, donde tiene lugar un sistema de interacciones sociopolíticas y económicas que producen un conjunto de relaciones interpersonales sobre la base de necesidades. Este sistema resulta portador de tradiciones, historia e identidad propias que se expresan en identificación de intereses y sentido de pertenencia que diferencian al grupo que integra dicho espacio de los restantes. (p. 172) 
Igualmente, dentro del contexto referencial, se hace alusión a los proyectos comunitarios, los cuales buscan abordar, de forma integral, una diversidad de asuntos e intereses existentes en la vida en sociedad del ser humano, no importando el lugar. Su participación va a permitir plantear proyectos comunitarios, los cuales son un:

Conjunto de acciones estratégicamente planificadas que involucran y articulan a personas interesadas en provocar cambios o trasformaciones en su realidad, para lo cual necesitan contar con una serie de recursos humanos y materiales, que utilizados racionalmente, les permiten producir bienes o servicios, de beneficio social, en un tiempo determinado, con un enfoque sostenible. (Centro de Intercambio y ReferenciaIniciativa Comunitaria -CIERIC-, 2005, p. 8)

Asimismo, los proyectos comunitarios son concebidos con la finalidad de: “(...) provocar un cambio o transformación de la realidad, (...) lograr satisfacer necesidades o mitigar, palear o solucionar problemas, (...) movilizar recursos, (...) alcanzar nuevas metas, (...) propiciar el desarrollo" (CIERIC, 2005, p. 8).

En este sentido, se resalta un aspecto fundamental para los proyectos de investigación y comunitarios: la evaluación, teniendo en cuenta que este es considerado por CIERIC (2005) como:

Un mecanismo que se realiza de una forma sistemática y dirigida; encaminada a identificar, obtener y proporcionar de manera confiable, datos e información suficiente y relevante, en qué apoyar un juicio acerca del mérito y el valor de los diferentes componentes de un proceso o de un conjunto de actividades específicas que se realizan con el propósito de producir efectos y resultados, comprobando la extensión y el grado en que dichos logros se han dado, de forma tal, que sirva de base o de guía para una toma de decisiones entre cursos de acción o para solucionar problemas o promover el conocimiento y la comprensión de los factores asociados al éxito o fracaso de sus resultados. (p. 41)

Igualmente, en una revisión realizada por Dannenberg (2016) se concluye que los principales impactos de las evaluaciones en salud revisadas influyeron en algunas decisiones, mejoraron la colaboración entre las partes interesadas, aumentaron la conciencia sobre los problemas de salud entre los tomadores de decisiones y dieron a los miembros de la comunidad una voz más fuerte en las decisiones locales.

Por otro lado, otros conceptos que se deben resaltar en el tema abordado son: promoción 
de la salud, prevención, relación entre promoción y prevención en la salud.

Para la OMS (como se citó en OPS, 2021), la promoción de la salud es:

El proceso que permite a las personas incrementar el control sobre su salud". Este concepto se pone en práctica usando enfoques participativos; los individuos, las organizaciones, las comunidades y las instituciones colaboran para crear condiciones que garanticen la salud y el bienestar para todos. (Párr. 1)

De otro lado, la OMS (como se citó en Fondo de Bienestar Social, 2013) define la prevención como las "Medidas destinadas no solamente a prevenir la aparición de la enfermedad, tales como la reducción de factores de riesgo, sino también a detener su avance y atenuar sus consecuencias una vez establecida" (Párr. 1).

Igualmente, el Ministerio de Salud (s.f.) relaciona los conceptos de promoción y prevención en salud de la siguiente manera: Proceso que proporciona a las poblaciones los medios necesarios para mejorar su salud, al igual que el ejercer sobre ella un mayor control, buscando encaminar las estrategias de intervención hacia los determinantes de la salud y la reducción de la inequidad; a través de la:

Formulación de política pública, creación de ambientes favorables a la salud, fortalecimiento de la acción y participación comunitaria, desarrollo de actitudes personales saludables y la reorientación de los servicios de salud; por sus características, la promoción de la salud supone una acción intersectorial sólida que hace posible la movilización social requerida para la transformación de las condiciones de salud. (párr. 1)

Por su parte, desde la profesión en fonoaudiología, los conceptos de promoción y prevención en salud son abordados desde el ámbito de la promoción de la salud comunicativa y la prevención de discapacidades de comunicación, cuyas competencias específicas profesionales los faculta para:

El diseño, ejecución y evaluación de planes, programas y proyectos orientados a generar procesos, dinámicas y estrategias saludables que faciliten el desarrollo, competencia y mediación entre grupos y personas para el logro del bienestar comunicativo. Estas acciones y procedimientos del saber hacer profesional del fonoaudiólogo, son desarrolladas en el primer y segundo nivel de atención en salud. (Ministerio de Salud et al., 2014). 


\section{Efecto de un programa | Revista Virtual \\ Universidad Católica del Norte, 65, 171-199 \\ ISSN: 0124-5821 (En línea)}

\section{Metodología}

El proyecto de investigación se llevó a cabo desde un enfoque cuantitativo, diseño no experimental, con un alcance descriptivo, longitudinal - retrospectivo. Se realizó un muestreo no probabilístico por conveniencia de todos los egresados y docentes coordinadores responsables de las actividades comunitarias de la zona, específicamente los que trabajaron el proyecto Salud Escolar, y docentes y administrativos de las instituciones educativas de la zona que asistían en la jornada matinal.

Teniendo en cuenta que los programas de proyección social que se desarrollan en la Universidad, en zonas vulnerables del municipio, son planeados para ser ejecutados durante un periodo no mayor de 5 años, para este estudio se tomó el período comprendido entre los años 20132017, teniendo en cuenta que en dicho tiempo la Facultad Ciencias de la Salud de la UNISUCRE hizo presencia en la zona suroriental del municipio de Sincelejo.

Para la selección de la muestra poblacional se tuvo en cuenta criterios de inclusión como: haber estado adscrito (para los docentes coordinadores) y matriculados (para los egresados) en el programa de Fonoaudiología de la Universidad de Sucre; igualmente, haber realizado actividades académicas comunitarias en las escuelas de la zona suroriental, estar vinculados al proyecto Salud Escolar en las escuelas de la zona, haber realizado actividades comunitarias en el proyecto Salud Escolar, durante los años 2013 al 2017, y diligenciar la encuesta (ya sea de forma virtual o física). Para docentes y administrativos de las instituciones educativas: pertenecer a las escuelas de la zona suroriental de Sincelejo, estar vinculado y participar activamente en el proyecto Salud Escolar; diligenciar la encuesta para docentes y administrativos proporcionada en medio físico, y pertenecer a la jornada matinal. Lo anterior, permitió que la muestra poblacional quedara conformada de la siguiente manera: 50 egresados del programa de Fonoaudiología, seis docentes, incluyendo un coordinador de las prácticas comunitarias del programa de Fonoaudiología, y 13 docentes y dos administrativos de las instituciones educativas de la zona de impacto del programa.

Para la recolección de la información se utilizó como instrumento de evaluación: la encuesta, en tanto para Valls-Llobet (2008) las encuestas de salud de población podrían ser un buen instrumento de observación de las necesidades, a este nivel, de la vida de las personas. En la investigación, para el diseño de esta, se utilizó el método de Likert, incluyendo preguntas cerradas 
con respuestas de tipo si - no y de selección. Las preguntas fueron elaboradas por los investigadores, teniendo en cuenta que permitieran establecer los niveles de impacto del programa, según los objetivos planteados. Igualmente, fueron diseñadas para ser respondidas de manera física y electrónica.

Durante el diseño de las encuestas se planeó organizar la información requerida en tres dimensiones, con sus respectivas variables; las dimensiones fueron las siguientes: efectividad del aprendizaje, capacidades de desempeño adquirido y actitud.

Para la primera dimensión se tuvo en cuenta las siguientes variables: porcentaje de cambio en los procesos enseñanza-aprendizaje, después de la participación del grupo de estudiantes de las instituciones educativas al proyecto; porcentaje de desempeño a nivel escolar del estudiante en la institución educativa, antes de vincularse al proyecto, y después de vinculado al proyecto; proceso comunicativo y de aprendizaje del estudiante antes de vincularse al proyecto y después de vinculado al proyecto.

Para la segunda dimensión, se tuvo en cuenta las variables: cambios presentados a nivel comunicativo, del lenguaje y escolar, de los estudiantes de la institución educativa, a partir de su participación en el proyecto; habilidades cognitivas, del lenguaje, aprendizaje y comunicativas, desarrolladas por los estudiantes después de participar en el proyecto. Finalmente, para la tercera dimensión: participación de los docentes de la institución educativa en el desarrollo del proyecto; interés de los docentes por los logros alcanzados por los niños a nivel comunicativo, del lenguaje y escolar; y contribución del docente en la reafirmación de los conocimientos y habilidades adquiridos por los niños, a partir de las actividades desarrolladas en el proyecto. Paralelo al diseño de las encuestas, se elaboró un cuestionario que permitiera a los investigadores identificar los profesionales externos con experticia y dominio en la realización de proyectos comunitarios, y poder ser seleccionados como jueces expertos para que evaluaran y validaran las encuestas realizadas, previo contacto con universidades del país que ofertaran los programas de fonoaudiología, como son Cauca, Bogotá y Cartagena.

Posteriormente, se procedió a validar las encuestas, al tener en cuenta cada uno de los aportes y criterios dados por los evaluadores. Luego, fueron aplicadas a egresados, docentes coordinadores de las prácticas comunitarias (de la UNISUCRE), docentes y coordinadores (de las instituciones educativas), teniendo en cuenta criterios personales, conocimiento de la ejecución del 
programa y voluntariedad de cada uno de los encuestados (Andréu, 2000).

Igualmente, para conocer la fiabilidad de las encuestas aplicadas, se calculó el coeficiente de Alpha de Cronbach (a), arrojando como resultado un a > 0,7, lo que permitió corroborar la fiabilidad de las encuestas elaboradas.

Otro instrumento de evaluación utilizado para la recolección de la información fue la revisión documental, la cual es:

Una técnica en donde se recolecta información escrita sobre determinado tema, teniendo en cuenta proporcionar variables que se relacionan indirecta o directamente con el tema establecido, vinculando estas relaciones, posturas o etapas, en donde se observe el estado actual de conocimiento sobre ese fenómeno o problemática existente. (Hurtado, como se citó en Núñez y Villamil, 2017, p. 9)

En la Facultad Ciencias de la Salud se viene compilando el trabajo realizado en la comunidad, a través de informes escritos que sustentan las actividades proyectadas durante las prácticas comunitarias por cada uno de los programas que la conforman (Fonoaudiología, Enfermería y Medicina). Para el presente proyecto, se hace revisión de los documentos del accionar fonoaudiológico en las comunidades (prácticas comunitarias), de los años 2013-2017.

Posteriormente, se realizó el análisis de la información, utilizando los estadísticos descriptivos para las variables numéricas y categóricas, datos procesados a través de la herramienta de análisis estadístico: IBM SPSS Statistics, versión 26.

Para el manejo de la información suministrada se tuvo en cuenta lo estipulado en la Resolución 8430 del 4 de octubre de 1993, título II de la Investigación en seres Humanos y la Declaración de Helsinki.

\section{Resultados}

Se dio alcance al primer objetivo específico, el cual buscaba establecer el grado de participación en el programa Salud Escolar de estudiantes y docentes de las instituciones educativas, estudiantes, egresados y docentes de la UNISUCRE, durante el período 2013- 2017, ya que, al revisar la documentación que soporta el proyecto Salud Escolar, del programa, se observó que son dos las escuelas de la zona Sur Oriental del municipio de Sincelejo (Escuelas La 
Unión y San José) que se vincularon al programa, centrándose la mayor parte del trabajo en la Institución Educativa La Unión, con un 67 \%. De los subproyectos implementados dentro del programa Salud Escolar (Juguemos con los sonidos, Leo-escribo y aprendo y Comuniquémonos mejor) se observa que la participación de los estudiantes de las instituciones educativas, para el 2013, en el programa Juguemos con los sonidos, fue de 348 estudiantes, el cual se incrementó para el 2017, con una partición de 438 estudiantes. En el programa: Leo-escribo y aprendo, para el 2013, se vincularon 181 estudiantes, con un mínimo incremento para el 2014, donde participaron 189 estudiantes; para los años posteriores, no se reportan actividades. El programa Comuniquémonos mejor vinculó para el 2013 a 900 estudiantes; sin reporte de actividades para los años posteriores. Se evidenció, entonces, que el programa que presenta permanencia en tiempo es Juguemos con los sonidos. En lo relacionado con número de egresados del programa de Fonoaudiología, por años, se pudo evidenciar que el 2014 fue el año en que más egresaron estudiantes del programa de Fonoaudiología (67 egresados), cifra que disminuyó para el 2017 en un $50 \%$. El programa siempre ha contado con un coordinador de prácticas comunitarias, y dos docentes que apoyan el proceso en la comunidad, como se puede observar en la Tabla 1 y Figura 1 .

\section{Tabla 1}

Estudiantes de las instituciones de educación de la zona Suroriental, vinculados al proyecto Salud Escolar durante los años 2013-2017

\begin{tabular}{lccccc}
\hline \multicolumn{1}{c}{ Subproyectos } & \multicolumn{5}{c}{ Años } \\
& 2013 & 2014 & 2015 & 2016 & 2017 \\
\cline { 2 - 6 } & & Número de estudiantes vinculados & \\
\hline Juguemos con los sonidos & 348 & 231 & 314 & 378 & 438 \\
\hline Leo-escribo y aprendo & 181 & 189 & - & - & - \\
\hline Comuniquémonos mejor & 900 & - & - & - & - \\
\hline
\end{tabular}

Nota: Elaboración propia. 


\section{Figura 1}

Actores docentes institución educativa y UNISUCRE - Fonoaudiólogos egresados durante los años 2013 a 2017
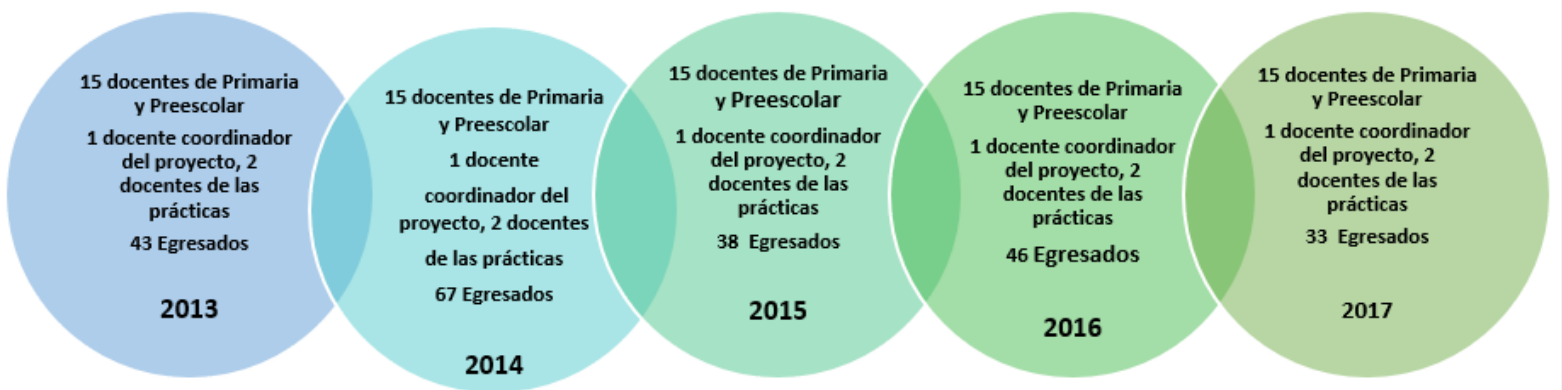

\section{ACTORES DEL PROYECTO}

Nota: Elaboración propia.

Por otro lado, se dio alcance al segundo objetivo específico: "Determinar el efecto a nivel de capacidades de desempeño adquiridas, a nivel cognitivo y comunicativo, de los niños y niñas involucrados al proyecto, de las instituciones educativas". Después de analizar los resultados arrojados del análisis de las variables categóricas SI y No, y de las respuestas obtenidas de las encuestas diligenciadas por egresados y docentes coordinadores de las prácticas del programa de Fonoaudiología, y docentes y coordinadores de las instituciones Educativas, se pudo evidenciar que tanto egresados como docentes coordinadores de las prácticas comunitarias coinciden al responder que las habilidades en las que mejor resultados obtienen los estudiantes vinculados al proyecto es a nivel de comunicación oral, conciencia fonológica y procesos de lectoescritura, con porcentajes reportados entre $50 \%$ y $100 \%$. Mientras que los docentes y administrativos de las instituciones educativas reportan otras habilidades, a nivel comunicativo, comprensión lectora, buenos hábitos orales y de autocuidado de la audición, con un porcentaje comprendido entre $60 \%$ y $100 \%$, como se puede observar en las tablas 2 y 3 . 


\section{Efecto de un}

programa

Efecto de un programa | Revista Virtual

Universidad Católica del Norte, 65, 171-199

ISSN: 0124-5821 (En línea)

\section{Tabla 2}

Dimensión capacidades de desempeño adquirido por los estudiantes de la institución educativa según egresados y docentes coordinadores de las prácticas comunitarias

\begin{tabular}{|c|c|c|c|c|}
\hline \multirow{3}{*}{ Variables } & \multicolumn{2}{|c|}{$\begin{array}{c}\text { Egresados } \\
\quad N=50\end{array}$} & \multicolumn{2}{|c|}{$\begin{array}{c}\text { Docentes coordinadores } \\
N=6\end{array}$} \\
\hline & & & & \\
\hline & Frecuencia & Porcentaje & Frecuencia & Porcentaje \\
\hline Buena comunicación oral & 28 & $56 \%$ & 4 & $66,7 \%$ \\
\hline Buena comunicación escrita & 30 & $60 \%$ & 2 & $33,3 \%$ \\
\hline Lectura fluida & 14 & $28 \%$ & 2 & $33,3 \%$ \\
\hline $\begin{array}{l}\text { Buena comprensión de lo leído y } \\
\text { escuchado }\end{array}$ & 14 & $28 \%$ & 2 & $33,3 \%$ \\
\hline Buenos hábitos orales & 16 & $32 \%$ & 2 & $33,3 \%$ \\
\hline $\begin{array}{l}\text { Producción correcta de los sonidos } \\
\text { del habla }\end{array}$ & 16 & $32 \%$ & 2 & $33,3 \%$ \\
\hline Cuidado de la audición y voz & 12 & $24 \%$ & 1 & $16,7 \%$ \\
\hline Comunicación efectiva & 4 & $8 \%$ & 5 & $83,3 \%$ \\
\hline Conciencia fonológica & 39 & $78 \%$ & 3 & $50 \%$ \\
\hline Hábitos orales adecuados & 6 & $12 \%$ & 1 & $16,7 \%$ \\
\hline Buena comprensión lectora & 18 & $36 \%$ & 1 & $16,7 \%$ \\
\hline Auto cuidado de la audición y voz & 7 & $14 \%$ & 1 & $16,7 \%$ \\
\hline $\begin{array}{l}\text { Reconocen agentes contaminantes } \\
\text { auditivos y orales }\end{array}$ & 7 & $14 \%$ & 2 & $33,3 \%$ \\
\hline $\begin{array}{l}\text { Utilización del punto y modo } \\
\text { articulatorio correcto de los } \\
\text { sonidos del habla }\end{array}$ & 15 & $30 \%$ & 4 & $66,7 \%$ \\
\hline $\begin{array}{l}\text { Reconocen que a cada fonema } \\
\text { corresponde un grafema }\end{array}$ & 17 & $34 \%$ & 6 & $100 \%$ \\
\hline $\begin{array}{l}\text { Facilidad para adquirir procesos de } \\
\text { lecto-escritura }\end{array}$ & 33 & $66 \%$ & 6 & $100 \%$ \\
\hline Comprenden textos escritos & 6 & $12 \%$ & 6 & $100 \%$ \\
\hline
\end{tabular}

Nota: Elaboración propia. 


\section{Tabla 3}

Dimensión capacidades de desempeño adquirido por los estudiantes de la institución educativa según docentes de la institución

\begin{tabular}{|c|c|c|}
\hline \multirow{3}{*}{ Variables } & \multicolumn{2}{|c|}{$\begin{array}{l}\text { Docentes de la institución educativa } \\
\qquad N=15\end{array}$} \\
\hline & & \\
\hline & Frecuencia & Porcentaje \\
\hline $\begin{array}{l}\text { Se produjo cambios a nivel comunicativo y } \\
\text { escolar en los involucrados en el proyecto }\end{array}$ & 15 & $100 \%$ \\
\hline Desarrollo de habilidades comunicativas & 13 & $86,6 \%$ \\
\hline $\begin{array}{l}\text { Desarrollo de habilidades de Conciencia } \\
\text { Fonológica }\end{array}$ & 13 & $86,6 \%$ \\
\hline Comprensión Lectora & 10 & $66,6 \%$ \\
\hline Buenos hábitos orales & 11 & $73,3 \%$ \\
\hline Autocuidado de la Audición & 9 & $60 \%$ \\
\hline
\end{tabular}

Fuente: Elaboración propia.

También, se dio alcance al tercer objetivo específico, el cual buscaba identificar el efecto en la actitud hacia procesos de aprendizaje, porcentajes de efectividad del aprendizaje y posibilidad de cambio en el desempeño de los involucrados en el proyecto. Primero, se realizó el análisis de variables numéricas en porcentajes (\%) de la dimensión efectividad del aprendizaje, de las encuestas diligenciadas por egresados, coordinadores de las prácticas comunitarias y docentes y administrativos de la institución educativa, encontrando que, en esta dimensión, los encuestados reportaron que hay dificultades de desempeño y habilidades escolares de los niños y niñas antes de vincularse al proyecto, con puntuaciones porcentuales comprendidas entre (máx. $=99 \% ; \mathrm{D}=$ 18,2) a (máx.=30\%; $\mathrm{D}=9,8 \%)$, mientras que después de vinculados al proyecto, estas puntuaciones porcentuales mejoraron, reportándose puntuaciones entre (máx.=95\%; $D=14,5 \%$ ) a (máx.=90\%; D= 10\%), como se puede observar en la Tabla 4. 


\section{Tabla 4}

Estadísticos descriptivos de la dimensión efectividad del aprendizaje

\begin{tabular}{|c|c|c|c|c|}
\hline \multicolumn{5}{|c|}{ Dimensión: Efectividad del aprendizaje } \\
\hline \multicolumn{5}{|c|}{ Egresados. $\mathbf{N}=\mathbf{5 0}$} \\
\hline \multicolumn{5}{|c|}{ Estadísticos descriptivos } \\
\hline Variables & $\begin{array}{l}\text { Míni } \\
\text { mo }\end{array}$ & $\begin{array}{l}\text { Máxi } \\
\text { mo }\end{array}$ & Media & Desviación \\
\hline $\begin{array}{l}\text { Dificultades presentadas por los estudiantes de la } \\
\text { institución educativa antes de integrarse al } \\
\text { proyecto (en \%) }\end{array}$ & $10 \%$ & $99 \%$ & $40,9 \%$ & $18,2 \%$ \\
\hline $\begin{array}{l}\text { Logros obtenidos por los estudiantes de la } \\
\text { institución educativa después de integrarse al } \\
\text { proyecto (en \%) }\end{array}$ & $20 \%$ & $95 \%$ & $75 \%$ & $13,6 \%$ \\
\hline Docentes coordinadores de las prácti & as Comuni & rias & & $=6$ \\
\hline \multicolumn{5}{|c|}{\begin{tabular}{|c|c|} 
Estadísticos descriptivos \\
\end{tabular}} \\
\hline Variables & $\begin{array}{l}\text { Míni } \\
\text { mo }\end{array}$ & $\begin{array}{l}\text { Máxi } \\
\text { mo }\end{array}$ & Media & Desviación \\
\hline $\begin{array}{l}\text { Dificultades presentadas por los estudiantes de la } \\
\text { institución educativa antes de integrarse al } \\
\text { proyecto (en \%) }\end{array}$ & $10 \%$ & $30 \%$ & $18,3 \%$ & $9,8 \%$ \\
\hline $\begin{array}{l}\text { Logros obtenidos por los estudiantes de la } \\
\text { institución educativa después de integrarse al } \\
\text { proyecto (en \%) }\end{array}$ & $70 \%$ & $90 \%$ & $83,3 \%$ & $10 \%$ \\
\hline \multicolumn{3}{|c|}{ Docentes y coordinador de la institución educativa } & \multicolumn{2}{|c|}{$\mathbf{N}=\mathbf{1 5}$} \\
\hline \multicolumn{5}{|c|}{ Estadísticos descriptivos } \\
\hline Variables & $\begin{array}{l}\text { Míni } \\
\text { mo }\end{array}$ & $\begin{array}{l}\text { Máxi } \\
\text { mo }\end{array}$ & Media & Desviación \\
\hline $\begin{array}{l}\text { Cambio en los procesos de Enseñanza } \\
\text { Aprendizaje (en \%) }\end{array}$ & $50 \%$ & $90 \%$ & $74 \%$ & $14,5 \%$ \\
\hline \multicolumn{5}{|c|}{ Frecuencias } \\
\hline & \multicolumn{2}{|c|}{ Bueno } & \multicolumn{2}{|c|}{ Regular } \\
\hline & $\begin{array}{l}\text { Frecue } \\
\text { ncia }\end{array}$ & $\begin{array}{l}\text { Porcen } \\
\text { taje }\end{array}$ & $\begin{array}{l}\text { Frecue } \\
\text { ncia }\end{array}$ & Porcentaje \\
\hline \multirow[t]{3}{*}{$\begin{array}{l}\text { Desempeño de los estudiantes antes de vincularse } \\
\text { al Programa }\end{array}$} & 1 & $6,6 \%$ & 14 & $93,3 \%$ \\
\hline & \multicolumn{2}{|c|}{ Bueno } & \multicolumn{2}{|c|}{ Excelente } \\
\hline & $\begin{array}{l}\text { Frecue } \\
\text { ncia }\end{array}$ & $\begin{array}{l}\text { Porcen } \\
\text { taje }\end{array}$ & $\begin{array}{l}\text { Frecue } \\
\text { ncia }\end{array}$ & Porcentaje \\
\hline $\begin{array}{l}\text { Desempeño de los estudiantes después de } \\
\text { vincularse al Programa }\end{array}$ & 13 & $86,6 \%$ & 2 & $13,3 \%$ \\
\hline
\end{tabular}

Nota: Elaboración propia. 
Por su parte, al analizar las variables categóricas SI y No, relacionadas con posibilidad de cambio en el desempeño de los involucrados en el proyecto ("actitud"), se observó que docentes y administrativos de las instituciones educativas reportan su participación, interés y apoyo en las actividades ejecutadas en el transcurso del proyecto, alcanzando puntuaciones porcentuales entre $60 \%$ y $73 \%$, como se observa en la Tabla 5 .

\section{Tabla 5}

Dimensión actitud de docentes y administrativos de las instituciones educativas

\begin{tabular}{lcccc}
\hline & \multicolumn{5}{c}{ Docentes y administrativos No 15 } \\
\cline { 2 - 5 } Variables & Frecuencia & Porcentaje & Frecuencia & Porcentaje \\
\cline { 2 - 5 } & & & 5 & $33 \%$ \\
\hline $\begin{array}{l}\text { Participación de docentes y } \\
\text { administrativos en el desarrollo del } \\
\text { proyecto }\end{array}$ & 10 & $67 \%$ & 4 & $27 \%$ \\
\hline $\begin{array}{l}\text { Interés de docentes y } \\
\text { administrativos por los logros } \\
\text { alcanzados por sus estudiantes }\end{array}$ & 11 & $73 \%$ & & \\
\hline $\begin{array}{l}\text { Apoyo de los docentes por } \\
\text { reafirmar conocimientos y } \\
\text { habilidades adquiridas durante } \\
\text { actividades académicas }\end{array}$ & 9 & $60 \%$ & 6 & $40 \%$ \\
\hline
\end{tabular}

Nota: Elaboración propia.

Al cruzar algunas variables de la dimensión "Capacidades de desempeño adquirido", y según los datos reportados por los egresados en las encuestas diligenciadas, se pudo observar que, de los 50 egresados, el $56 \%$ respondió que los estudiantes que participaron en el proyecto Sí alcanzaron una buena comunicación oral; de estos, el 64,3 \% respondió que Sí alcanzó una buena comunicación escrita. Al cruzar la variable buenos hábitos orales y producción correcta de los sonidos del habla con buena comprensión de lo escuchado y lo leído, se observó que, de los 50 egresados, el 32 \% respondió que Sí alcanzó buenos hábitos orales y producción correcta de los sonidos del habla; de estos, el 56,3 \% respondió que Sí alcanzó una buena comprensión de lo leído y lo escuchado. Al cruzar las variables conciencia fonológica y comunicación efectiva, se observó que, de los 50 egresados, el 78 \% respondió que Sí alcanzó una comunicación efectiva; de estos, el 5,1 \% Sí alcanzó la conciencia fonológica (ver Tabla 6) 


\section{Tabla 6}

Cruce entre algunas variables de la dimensión capacidades de desempeño adquirido

\begin{tabular}{|c|c|c|c|c|c|}
\hline \multicolumn{6}{|c|}{ Tabla Cruzada Buena comunicación oral con buena comunicación escrita } \\
\hline & & & $\begin{array}{r}\text { Buena c } \\
\text { esc }\end{array}$ & cación & Total \\
\hline & & & No & $\mathbf{S i}$ & \\
\hline \multirow{6}{*}{$\begin{array}{c}\text { Buena comunicación } \\
\text { oral }\end{array}$} & \multirow{3}{*}{ No } & Recuento & 10 & 12 & 22 \\
\hline & & $\begin{array}{l}\text { \%buena Com. } \\
\text { Oral }\end{array}$ & $45,5 \%$ & $54,5 \%$ & \\
\hline & & $\begin{array}{l}\text { \% buena Com. } \\
\text { Escrita }\end{array}$ & & & $44 \%$ \\
\hline & \multirow{3}{*}{ Si } & Recuento & 10 & 18 & 28 \\
\hline & & $\begin{array}{l}\text { \%buena Com. } \\
\text { Oral }\end{array}$ & $35,7 \%$ & $64,3 \%$ & \\
\hline & & $\begin{array}{l}\text { \% buena Com. } \\
\text { Escrita }\end{array}$ & & & $56 \%$ \\
\hline \multirow{3}{*}{ Total } & & Recuento & 20 & 30 & 50 \\
\hline & & $\begin{array}{l}\text { \%buena Com. } \\
\text { Oral }\end{array}$ & $40 \%$ & $60 \%$ & $100 \%$ \\
\hline & & $\begin{array}{l}\% \text { buena Com. } \\
\text { Escrita }\end{array}$ & $100 \%$ & $100 \%$ & $100 \%$ \\
\hline
\end{tabular}

Tabla cruzada Buenos hábitos orales y producción correcta de los sonidos del habla con buena comprensión de lo leído y escuchado

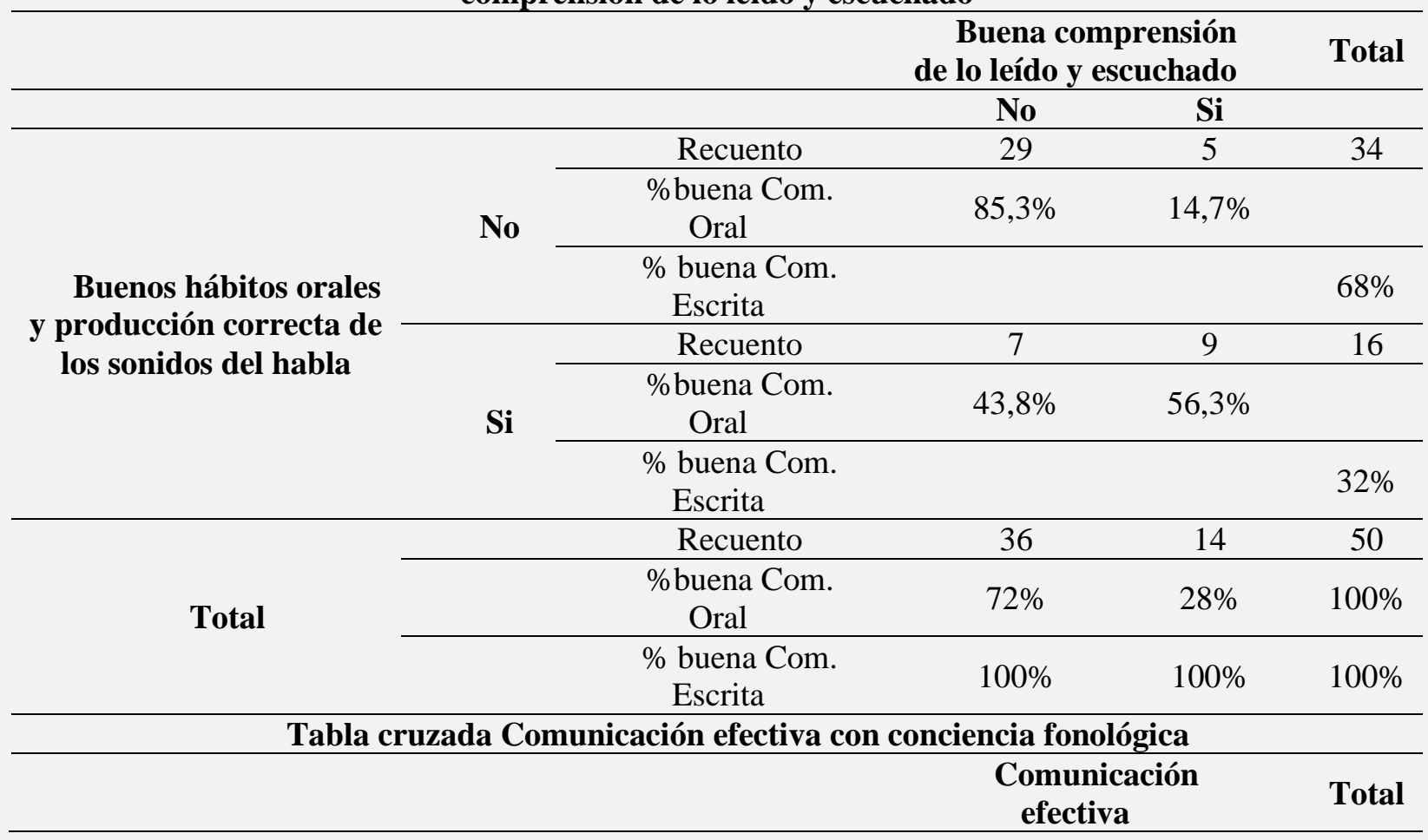




\begin{tabular}{|c|c|c|c|c|c|}
\hline & & & No & $\mathbf{S i}$ & \\
\hline \multirow{6}{*}{ Conciencia fonológica } & \multirow{3}{*}{ No } & Recuento & 9 & 2 & 11 \\
\hline & & $\begin{array}{l}\text { \%buena Com. } \\
\text { Oral }\end{array}$ & $81,8 \%$ & $18,2 \%$ & \\
\hline & & $\begin{array}{l}\text { \% buena Com. } \\
\text { Escrita }\end{array}$ & & & $22 \%$ \\
\hline & \multirow{4}{*}{$\mathbf{S i}$} & Recuento & 37 & 2 & 39 \\
\hline & & $\begin{array}{l}\text { \%buena Com. } \\
\text { Oral }\end{array}$ & & & \\
\hline & & $\begin{array}{l}\text { \% buena Com. } \\
\text { Escrita }\end{array}$ & & & $78 \%$ \\
\hline Total & & Recuento & 46 & 4 & 50 \\
\hline
\end{tabular}

Nota: Elaboración propia

\section{Discusión}

Santos (2005) manifiesta que es necesario comprender la salud desde un planteamiento innovador, el cual permita llegar al ámbito escolar, para así mejorar las acciones que se orientan a promover estilos de vida saludables.

Partiendo de esta premisa, el programa de Fonoaudiología de la UNISUCRE busca disminuir factores de riesgo en la población de diferentes grupos etarios y condiciones psicosociales, para evitar a futuro afecciones, a nivel comunicativo, lenguaje, cognitivo y auditivo, a través de acciones de promoción y prevención de la salud (a nivel primario y secundario). Es así que, desde las prácticas comunitarias, se viene haciendo presencia en las comunidades más vulnerables del municipio de Sincelejo, con el objeto de implementar programas dirigidos a proporcionar el bienestar comunicativo e integral del individuo; este accionar es abordado desde el contexto escolar, ya que es este el espacio considerado como ese primer y mejor momento para desarrollar y adquirir estilos de vida saludable (Cantera y Devís, 2002), hecho que invita a las instituciones educativas a estar preparadas para los cambios que se generan a partir de algunas situaciones, expectativas e inconvenientes que se generan a partir de los cambios propios de una sociedad (Ahrabi-Fard y Matvienko, 2005).

Al analizar los datos obtenidos en el estudio, se pudo evidenciar que los resultados que dan alcance al primer objetivo específico concuerdan con lo expuesto por Zapata y Rondán (2016), quienes exponen que, para crear un enfoque participativo, se requiere que la persona asuma una actitud activa y participativa, en acciones que involucran programas y proyectos de salud, en pro 
de mejorar y satisfacer necesidades de una comunidad particular y poder vivir bien. Asimismo, la realización del estudio confirma lo reportado por Correa y Bedoya (2010), en su investigación, en la cual concluyen que "la estrategia de reducción, orientada a la promoción, prevención e intervención del riesgo de salud en las personas, familias y comunidades, es la más utilizada por las universidades apoyada en sus procesos investigativos" (p. 142). Igualmente, generó conocimientos relacionados con el impacto ejercido por los programas implementados en contextos educativos, y que se relacionan con el quehacer fonoaudiológico, fortaleciendo la identidad de la UNISUCRE por su labor en contextos vulnerables y reconocimiento por su abordaje integral. En este sentido, se da cumplimiento a una de las metas propuestas en el plan de mejora del programa de Fonoaudiología, la cual tiene que ver con el diseño y ejecución de proyectos que permitan visualizar los programas bandera que identifican a la Facultad, y que se vienen desarrollando en las comunidades más vulnerables del municipio, dentro de sus actividades comunitarias.

Por otro lado, los resultados obtenidos dan alcance a los objetivos dos y tres, en lo relacionado con efectividad del aprendizaje y capacidad de desempeño adquirido, sustentándose desde la teoría, la cual puntualiza que: es en la escuela primaria donde se dan las condiciones adecuadas para el trabajo con niños y niñas, dado a que se encuentran en una edad propicia para recibir la intervención de profesionales, pasando el docente, a convertirse en agente protector, teniendo en cuenta su cercanía con niños y niñas (Hernández et al., 2015, p. 248).

Investigaciones recientes dan cuenta de la importancia de abordar y estimular tempranamente al infante en el desarrollo y adquisición de sus habilidades y destrezas, necesarias para el aprendizaje escolar, ya que se ha evidenciado que la gran mayoría de las dificultades o deficiencias presentadas en los procesos de adquisición de la lectura, en los primeros años de educación, se presentan por estar asociados a un problema de base, el cual interfiere de una manera u otra en el normal desarrollo de los precurrentes necesarios para la adecuada adquisición de habilidades lectoras, y que se visibilizan cuando el menor ingresa a la escuela (Knight \& Gilpatrick, 2019). Con lo anteriormente expuesto, se puede afirmar que, al ejecutar proyectos de intervención, que favorezcan habilidades y competencias cognitivas-comunicativas y lingüísticas en poblaciones escolares más desfavorables, se está contribuyendo a proporcionar entornos 
educativos más saludables y enriquecedores, los cuales conducen a una adecuada adquisición de aprendizajes en los niños.

Es necesario indicar que, al cruzar algunas variables del estudio, se observó que los resultados obtenidos concuerdan con lo expuesto por Flórez (2004), quien da a conocer la importancia de reconocer la relación estrecha entre el lenguaje y la educación, ya que es el lenguaje esa herramienta comunicativa del ser humano que le va a permitir comprender saberes y valores, facilitando así el acceso al conocimiento y a la cultura, y generando transformaciones en su propio mundo e identidad. Flórez (2004) da un gran valor al lenguaje oral, ya sea en momentos iniciales o en las etapas más tardías de adquisición de los procesos lectores. Igualmente, autores como Difabio (2005), De Mier et al. (2012), Florit \& Levorato (2012), entre otros, han demostrado la estrecha relación causal entre variables como vocabulario y comprensión.

\section{Conclusiones}

Se puede concluir que el proyecto comunitario Salud Escolar contribuye a mejorar indicadores comunicativos y de aprendizaje en la población educativa de la zona, como también se evidenció una buena aceptación por parte de la comunidad educativa (docentes, estudiantes y administrativos), en lo relacionado con el desarrollo y mantenimiento de actividades durante períodos largos. Esto lleva a afirmar que la implementación del programa Salud Escolar pone en manifiesto que, en las instituciones educativas, el desarrollo de las prácticas comunitarias da cumplimiento a las necesidades comunicativas y del lenguaje, al igual que ratifica que se están realizando los procesos adecuados en la intervención que se le ofrece a los estudiantes.

Por otro lado, la ejecución del proyecto contribuye con el aporte de un estudio con enfoque preventivo, que aborda tempranamente el desarrollo de habilidades cognitivas, lenguaje y comunicación, proporcionando entornos educativos saludables. Lo anterior, teniendo en cuenta que, al desarrollar de forma sólida y temprana el lenguaje y la comunicación de los niños, se les está preparando para la adquisición de aprendizajes escolares, de manera que comprendan textos escritos, adquieran un mejor rendimiento académico y mejores resultados ocupacionales en el futuro. 
Igualmente, es posible afirmar que el programa de Fonoaudiología de la UNISUCRE ha ofrecido a sus estudiantes una formación adecuada, la cual les permite, a través de las experiencias vividas en entornos comunitarios, integrar el saber con el hacer profesional de manera exitosa, e igualmente, el impactar positivamente en las comunidades vulnerables. Sin embargo, se debe considerar que existen algunos aspectos a mejorar dentro de las actividades desarrolladas, como evaluar en tiempos más cortos el impacto que se está ejerciendo en las comunidades, con la implementación de los proyectos. Igualmente, antes de implementar los proyectos, realizar un diagnóstico inicial que dé cuenta de cómo se encuentran las comunidades antes de su vinculación, lo cual permita visualizar cómo se van produciendo cambios.

En los documentos que reposan en la Facultad Ciencias de la Salud y en el programa de Fonoaudiología, producto del trabajo realizado durante todos los años en que el programa hizo presencia en la zona Suroriental, no se consiguieron reportes que vincularan a entes estatales ni gubernamentales, microempresas ni empresas privadas de gran envergadura de la región, en acciones tendientes al desarrollo de habilidades comunicativas, lenguaje y aprendizaje con las comunidades atendidas.

Para finalizar, se plantea la necesidad de establecer alianzas que permitan la difusión y consolidación del conocimiento que esta práctica produce; igualmente, materializar nuevas investigaciones con los diferentes proyectos que se vienen implementando a nivel comunitario en la Facultad. Asimismo, involucrar a la familia en los programas desarrollados con escolares.

\section{Referencias}

Ahrabi-Fard, I., y Matvienko, O. (2005). Promoción de una educación activa de la actividad física orientada a la salud en las clases de educación física. Cultura, Ciencia y Deporte, 1(3) ,163170. https://www.redalyc.org/articulo.oa?id=163017986008

Andréu, J. (2000). Las técnicas de análisis de contenido: una revisión actualizada. http://mastor.cl/blog/wp-content/uploads/2018/02/Andreu.-analisis-de-contenido.-34pags-pdf.pdf

Arredondo Kassabb, M. (2019). Informe de resultados e impacto social del proyecto "Buenas prácticas socioeducativas comunitarias para la atención logopédica en la comunicación y 
el lenguaje en niños, escolares adolescentes, jóvenes y adultos". https://www.researchgate.net/publication/331839601_atencion_logopedica_comunitaria

Bennasar, M. (2011). Estilo de vida y salud en estudiantes universitarios: la universidad como entorno promotor de la salud [Tesis de doctorado]. Universidad de Illes Balears. http://www.academia.edu/6223906/estilos_de_vida_y_salud_en_estudiantes_universitari os_la_universidad_como_entorno_promotor_de_la_salud._miguel_bennasar_veny

Cantera, M., y Devís, J. (2002). La promoción de la actividad física relacionada con la salud en el ámbito escolar. Implicaciones y propuestas a partir de un estudio realizado entre adolescentes. Educación Física y Deportes, (67), 54-63. https://dialnet.unirioja.es/servlet/articulo?codigo $=2428188$

Castro, M., y Morales, M. (2015). Los ambientes de aula que promueven el aprendizaje, desde la perspectiva de los niños y niñas escolares. Revista Electrónica Educare, 19(3), 1-32. http://dx.doi.org/10.15359/ree.19-3.11

Carreño Patiño, L. B. (2018). Acciones del fonoaudiólogo(a) en el marco de la atención primaria de salud (APS); la teoría frente a la práctica [Trabajo de grado de especialización]. Universidad Nacional de Colombia. https://bit.ly/3m0QVrL

Centro de Intercambio y Referencia-Iniciativa Comunitaria. (2005). Propuesta metodológica para la gestión de proyectos. http://www.ciericgp.org/sites/default/files/propuesta_metodologica_para_la_gestion_de_ proyectos.pdf

Consejo de Facultad Ciencias de la Salud, Universidad de Sucre. (11 de junio de 2019). Resolución Número 022, por medio de la cual se avala y recomienda ante la DIUS la ejecución del Proyecto de investigación "Experiencias Académicas del Programa de Fonoaudiología de la UNISUCRE en Practicas Comunitarias de la zona Suroriental de Sincelejo, durante el periodo 2013-2017"'.

Correa, J. I., y Bedoya, M. (febrero-mayo, 2010). Intervención en el manejo social del riesgo en infancia y adolescencia. Revista Virtual Universidad Católica del Norte, (29), 122-144. https://revistavirtual.ucn.edu.co/index.php/RevistaUCN/article/view/74

Cortés, M., Leyva, C., y Organista, D. (2017). Comprensión de las Prácticas Fonoaudiológicas realizadas por egresados del programa de Fonoaudiología de la Corporación 
Universitaria Iberoamericana con la aplicación del Modelo Sistémico de la Comunicación [Trabajo de grado de pregrado]. Corporación Universitaria Iberoamericana. https://bit.ly/3oP3Rm8

Dannenberg, A. (2016). Effectiveness of Health Impact Assessments: A Synthesis of Data From Five Impact Evaluation Reports [Efectividad de las evaluaciones de impacto en la salud: síntesis de datos de cinco informes de evaluación de impacto]. Preventing Chronic Disease, 13. http://dx.doi.org/10.5888/pcd13.150559

De Mier, M., Borzone, A. M., y Cupani, M. (2012). La fluidez lectora en los primeros grados: relación entre habilidades de decodificación, características textuales y comprensión. Un estudio piloto con niños hablantes de español. Revista Neuropsicología Latinoamericana, 4(1), 18-33. https://www.redalyc.org/pdf/4395/439542720003.pdf

Difabio, H. (2005). Competencias para la comprensión de textos y el pensamiento crítico en nivel medio y universitario [Tesis de doctorado]. Universidad Nacional. https://bdigital.uncu.edu.ar/objetos_digitales/4624/difabiocompcomprtextnivuniv.pdf

Dockrell, J. E., \& Marshall, C. R. (2014). Measurement Issues: Assessing language skills in young children [Cuestiones de medición: evaluación de las habilidades lingüísticas en niños pequeños]. Child and Adolescent Mental Health, 20(2), 116-125. https://doi.org/10.1111/camh.12072

Flórez, R. (2004). El lenguaje en la educación. Una perspectiva fonoaudiológica. Universidad Nacional de Colombia.

Florián, P. (2019). Efectos del Programa de Educación en Salud en la Formación de Hábitos Saludables en los Docentes de Primaria, Institución educativa Indoamericano Rio Seco, El Porvenir. Revista Ciencia y Tecnología, 15(4), 121-132. https://revistas.unitru.edu.pe/index.php/PGM/article/view/2671\#: :text=Se\%20obtuvo\%2 0como\%20conclusi\%C3\%B3n\%20que,0\%2C0095\%20en\%20h\%C3\%A1bitos\%20saluda bles

Florit, E., \& Levorato, M. C. (2012). A longitudinal study on listening text comprehension and receptive vocabulary in preschoolers [Un estudio longitudinal sobre la comprensión auditiva de textos y el vocabulario receptivo en niños en edad preescolar]. Rivista di Psicolinguistica Applicata, 1/2(12), 65-80. https://bit.ly/3GDr9BA 
Efecto de un

programa
Efecto de un programa | Revista Virtual Universidad Católica del Norte, 65, 171-199

ISSN: 0124-5821 (En línea)

Fondo de Bienestar Social. (2013). Prevención de la enfermedad. https://www.fbscgr.gov.co/index.php?idcategoria=3906\#: :text=La\%20prevenci\%C3\%B 3n\%20de\%201a\%20enfermedad,sus\%20consecuencias\%20una\%20vez\%20establecida

Gobierno de Colombia. (s.f.). Política Nacional de Infancia y Adolescencia (2018-2030). https://www.icbf.gov.co/sites/default/files/politica_nacional_de_infancia_y_adolescencia 2018 _2030.pdf

Henwood, T., Channon, S., Penny, H., Robling, M., \& Waters, S. (2020). Do home visiting programmes improve children's language development? A systematic review [¿Los programas de visitas domiciliarias mejoran el desarrollo del lenguaje de los niños? Una revisión sistemática]. International Journal of Nursing Studies,109, Artículo 103610. https://doi.org/10.1016/j.ijnurstu.2020.103610

Hernández, E., Hernández, V., y Águila, Y. (mayo-junio, 2015). La promoción de la salud en el ámbito escolar mediante la prevención de adicciones. Revista Médica Electrónica, 37(3), 246-262. http://scielo.sld.cu/scielo.php?script=sci_arttext\&pid=S1684-

\section{$\underline{18242015000300007}$}

Knight, S., \& Gilpatrick, M. (2019). ELL Families: Myths, Truths and Strategies for Engagement [Familias ELL: mitos, verdades y estrategias para la participación]. Journal of English Learner Education, $\quad 9(1), \quad 1-6$. https://stars.library.ucf.edu/cgi/viewcontent.cgi?article $=1000 \&$ context=jele

$\begin{array}{lllll}\text { Ministerio de } & \text { Salud. } & \text { (s.f.). Promoción } & \text { y prevención. }\end{array}$ https://www.minsalud.gov.co/salud/Paginas/Promoci\%C3\%B3nyPrevenci\%C3\%B3n.asp $\underline{\mathrm{x}}$

Ministerio de Salud. (4 de octubre de 1993). Resolución 8430, Por la cual se establecen las normas científicas, técnicas y administrativas para la investigación en salud. https://www.minsalud.gov.co/sites/rid/Lists/BibliotecaDigital/RIDE/DE/DIJ/RESOLUCI ON-8430-DE-1993.PDF

Ministerio de Salud, ASOFON, ASOFONO, Colegio Colombiano de Fonoaudiología y ASOAUDIO. (2014). Perfil y competencias Profesionales del Fonoaudiólogo en Colombia. 
https://www.minsalud.gov.co/sites/rid/Lists/BibliotecaDigital/RIDE/VS/TH/Fonoaudiolo gia_Octubre_2014.pdf

Núñez, W., y Villamil, L. (2017). Revisión documental: el estado actual de las investigaciones desarrolladas sobre empatía en niñas y niños en las edades comprendidas entre los 6 a 12 años de edad surgidas en países latinoamericanos de habla hispana, entre los años 2010 al primer trimestre del 2017 [Trabajo de grado de pregrado]. Universidad Minuto de Dios. https://repository.uniminuto.edu/jspui/bitstream/10656/5218/1/TP_NunezMeraWendyJoh anna_2017.pdf

Organización Panamericana de la Salud. (2021). Promoción de la Salud. https://www.paho.org/es/temas/promocion-salud

Organización Panamericana de la Salud, y Organización Mundial de la Salud. (2010). Redes Integradas de Servicios de Salud: Conceptos, Opciones de Política y Hoja de Ruta para su Implementación en las Américas.

OPS. https://www.paho.org/uru/dmdocuments/RISS\%202010-

Doc\%20Posicion\%20revisado\%20pos\%20CD.pdf

Ramos, J., Álvarez, V., Tovaruela, N., Mahillo, R., y Gago, F. (marzo-abril, 2016). Impacto poblacional de un programa de salud escolar podológica. Gaceta Sanitaria, 30(2), 137-138. https://scielo.isciii.es/pdf/gs/v30n2/nota_campo1.pdf

Reyes, A. E., y Góngora, M. (2010). El trabajo comunitario integrado: Su aplicación en el trabajo social. Revista Humanismo y Trabajo Social, 9, 171-189. https://buleria.unileon.es/bitstream/handle/10612/3253/Angel.pdf?sequence=1\&isAllowe $\underline{\mathrm{d}=\mathrm{y}}$

Santos, S. (2005). La Educación Física escolar ante el problema de la obesidad y el sobrepeso. Revista Internacional de Medicina y Ciencias de la Actividad Física y el Deporte, 5(19), 179-199. http://cdeporte.rediris.es/revista/revista19/artobesidad10.htm

Silva, A., Escudero, P., Hidalgo, R., y Del Campo, M. (mayo-agosto, 2018). Estudio Cualitativo de la Práctica Fonoaudiológica en el Contexto de la Atención Primaria de la Salud, en la Quinta Región de Valparaíso. Ciencia y Trabajo, 20(62), 103-106. https://www.scielo.cl/scielo.php?script=sci_arttext\&pid=S0718-24492018000200103 


\section{Efecto de un}

Valls-Llobet, C. (2008). Salud comunitaria con perspectiva de género. Revista Comunidad, 10, 6170. https://pacap.net/es/publicaciones/pdf/comunidad/10/documentos3.pdf

Villarreal, A. (2012). Programa de Extensión y Proyección Social. "Una apuesta a la atención Primaria en Salud". Universidad de Sucre.

Waldfogel, J., \& Washbrook, E. V. (2010). Low income and early cognitive development in the UK: A report for the Sutton Trust [Bajos ingresos y desarrollo cognitivo temprano en el Reino Unido: un informe para Sutton Trust]. University of BRISTOL. https://core.ac.uk/download/pdf/29026856.pdf

Zapata, F., y Rondán, V. (2016). La Investigación Acción Participativa: Guía conceptual y metodológica del Instituto de Montaña. Instituto de Montaña. https://mountain.pe/recursos/attachments/article/168/Investigacion-Accion-ParticipativaIAP-Zapata-y-Rondan.pdf 